\title{
Adolescentes de género femenino: víctimas de maltrato infantil en El Salvador
}

\section{Female adolescents: child abuse victims in El Salvador}

\section{ISSN 2071-8748 \\ E-ISSN 2218-3345 \\ (c) (1) $\$$ \\ BY NC SA}

\author{
David Quintana \\ (ORCID) 0000-0002-6690-2360 \\ davidquintana@uls.edu.sv \\ Recibido: 3 de abril 2019 \\ Aprobado: 22 de agosto 2019
}

DOI: https://doi.org/10.5377/entorno.v0i68.8458

URI: http://hdl.handle.net/11298/1138

\section{Resumen}

Diversos estudios indican que en El Salvador, la violencia, no solo no disminuye, sino que por el contrario aumenta, a pesar que el Estado, señala que: "las niñas, niños y adolescentes deben ser tratados con respeto y no ser sometidos a castigos corporales, psicológicos o cualquier otro trato ofensivo (...)." (UTE, 2011, pág. 20).

El estudio realizado en el marco del proyecto denominado "Fortaleciendo la Colaboración en las Américas sobre Derechos de la Niñez y la Adolescencia" (por sus siglas en inglés RCYP), tuvo como objetivo identificar situaciones de maltrato infantil en adolescentes entre 13-15 años de edad.

La investigación fue realizada, bajo el paradigma cuantitativo, propio del método deductivo, y desde un enfoque de género, a partir del análisis de las mediciones sobre diferentes formas de maltrato hacia la adolescencia. En congruencia con el método, se utilizó la técnica de

\section{Abstract}

Numerous studies indicate that violence in El Salvador not only decreases, but that, on the contrary, it also increases regardless of what the State points out: "girls, boys and adolescents must be treated with respect, and must not be subjected to physical or psychological punishments, nor to any other form of abuse (...)." (UTE, 2011, p. 20)

In this sense, the objective of this study, was to identify child abuse situations in adolescents ranging between the ages of 13 and 15 . The study was conducted within the framework of the project "Fortaleciendo la Colaboración en las Américas sobre Derechos de la Niñez y la Adolescencia" (Rights for Children and Youth Partnership, RCYP)

The research was conducted under the quantitative paradigm, typical of the deductive method, and from a gender perspective, based on the analysis of the measurements made to the different forms of abuse

1 Miembro de la Unidad de Investigación de la Universidad Luterana Salvadoreña (ULS). Maestro en Métodos y Técnicas de Investigación Social. Licenciado en Ciencias de la Educación. Profesor de la cátedra de Metodología de la Investigación y Estadística Social. 
la encuesta, a través de un cuestionario aplicado a 384 adolescentes seleccionados mediante un muestreo probabilístico, en las tres zonas del país (Occidental, Central y Oriental),

El estudio indica, que el maltrato infantil, continúa siendo una forma de violencia ejercida con mayor frecuencia, contra las mujeres, puesto que, durante el año 2018, dos de cada tres adolescentes golpeados, resultaron ser de género femenino.

\section{Palabras clave}

Violencia infantil - El Salvador. Abuso del niño - El Salvador. Problemas sociales - El Salvador.

\section{Introducción}

\subsection{Marco contextual de la violencia}

En términos generales, se considera que "la violencia es un fenómeno multidimensional que puede ser de diverso tipo (física, psicológica, patrimonial, política, social, institucional, cultural, moral, emocional y sexual) y se puede dar en distintos ámbitos (familiar, institucional, laboral, etc.)" (Unicef El Salvador, 2014, p. 37).

Se estima que su origen obedece a múltiples causas que se manifiestan en el fortalecimiento de relaciones estructurales de desigualdad en los ámbitos político, económico y social; "económico, en el sentido que, si bien no la determina directamente, exacerba la vulnerabilidad de la niñez y adolescencia a tener sus derechos violentados (...)" (Unicef El Salvador, 2014, p. 20). Aunque, según la EHPM (2013) el nivel de pobreza por ingresos se ha reducido en los últimos años, todavía afecta al 34,8 \% de los hogares salvadoreños.

Acorde con Unicef El Salvador (2014, p. 37), entre sus principales causas figuran "la existencia de un entorno social y económico deteriorado, la ausencia de oportunidades de desarrollo humano, la carencia de espacios apropiados para la recreación, el debilitamiento de la institucionalidad pública y de la familia ocasionado por la desintegración familiar, el vacío de autoridad competente, la impunidad y towards adolescents. A survey was used in congruency to this method; the questionnaire was conducted with 384 adolescents who were selected via a probabilistic sampling, in the three areas of the country (western, central and eastern).

This study points out that child abuse continues to be a form of violence more frequently exerted against women, since during 2018 two out of three beaten adolescents turned out to be female.

\section{Keywords}

Child violence - El Salvador. Child abuse - El Salvador. Social problems - El Salvador.

la debilidad de los procesos de investigación y persecución del delito". Situación que, se considera, ha propiciado el aumento a niveles históricos de la violencia en el país.

El fenómeno de la violencia, no constituye algo nuevo, puesto que "El Salvador ha vivido una cultura autoritaria y de violencia a lo largo de su historia" (Unicef El Salvador, 2014, p. 18). La administración estatal durante décadas a cargo de dictaduras militares, el estallido de la guerra civil, la proliferación de maras y pandillas, constituyen evidencia de ello. Puesto que "ha prevalecido un Estado alejado de la población, que organiza la legislación y la política pública al margen de la sociedad civil y sin considerar las diferentes demandas y necesidades de la población". Muestra de esta situación es que la inversión específica en áreas como salud, educación, vivienda y seguridad ciudadana, que son servicios básicos para la subsistencia y desarrollo digno de la población, es todavía insuficiente.

Como consecuencia de este abandono estatal, según la publicación oficial de Unicef en el informe "Ocultos a plena luz" correspondiente al año 2014, El Salvador fue considerado el país con la tasa más alta del mundo de homicidios en menores de 19 años, con 27 por cada 100.000 habitantes, lo cual pone en evidencia "el alto nivel de violencia en el que vive el país (...) situación que atenta contra el pleno ejercicio de los derechos de niñas, niños, y adolescentes" (Unicef El Salvador, 2014, p. 20). 
Innumerables estudios indican la existencia de altos niveles de violencia experimentados por la niñez y la adolescencia. Se estima que las prácticas de crianza realizadas por parte de padres, madres y/o cuidadores ha construido un andamiaje cultural que, en gran medida, sostiene lo que "se trata de un problema alimentado por las normas sociales que toleran la violencia, al considerarla una manera aceptable de resolver los conflictos, además de aprobar la dominación de los niños por parte de los adultos." (Unicef, S.f, p. 6)

Esta situación confirma, según Unicef, S.f (p. 6), que "si un niño sufre castigos violentos a manos de sus padres $u$ otras personas que le cuidan, se considera que se trata de un hecho sin importancia y [lo más perjudicial es que] el menor no recibe protección judicial similar a la que se da a los adultos". Lamentablemente, esa falta de protección, combinada con las actitudes y normas sociales que justifican los actos de violencia contra la niñez, crea un entorno en el que muchas formas de violencia se consideran normales y finalmente quedan en la impunidad.

Conforme estos escenarios, el silencio, la negación y naturalización hacia las diferentes formas de violencia terminan por consolidarse como un mecanismo de autodefensa, según lo describe Unicef (S.f,) a continuación.

Independientemente del tipo de violencia que hayan sufrido o las circunstancias en que ésta se haya producido, la mayoría de las víctimas la mantiene en secreto y no solicita ayuda. (...) [Un aspecto a resaltar es que por ejemplo] casi la mitad de todas las niñas adolescentes de 15 a 19 años que mencionaron haber sido objeto de violencia física o sexual también dijeron que nunca se lo habían contado a nadie (p. 5).

Aunado a lo anterior, "una de las limitaciones propias de todo intento de documentar la violencia contra los niños es que no se contempla a un gran número de niños que no puede o no quiere denunciar sus experiencias". (Unicef, S.f, p. 6). Es decir, ante estos escenarios, los marcos muestrales utilizados en la medición de la violencia regularmente suelen ser limitados.

Sin embargo, en materia legislativa, se han desarrollado diferentes esfuerzos que evidencian el interés en la protección de los derechos de la niñez y adolescencia, siendo este el caso de la conformación del Sistema de Protección Integral de la Niñez y Adolescencia, el Instituto Salvadoreño para el Desarrollo Integral de la Niñez y Adolescencia, la Ley de Protección Integral de la Niñez y Adolescencia, el Consejo Nacional de la Niñez y de la Adolescencia (Conna), las Juntas de Protección a escala departamental, entre otros (Unicef El Salvador, 2014).

Por otra parte, en términos estadísticos, la distribución por sexo de la adolescencia es relativamente equilibrada, ya que el $51,2 \%$ (1.126.329) es femenina y el $48,8 \%$ (1.075.478) masculina, según los datos de la EHPM (2013). En términos de pobreza, se estima que un $43,7 \%$ del total $\left(\mathrm{NNA}^{2}\right.$ ) reside en hogares pobres, de los cuales el $31,5 \%$ se encuentran en condición de pobreza relativa y $12,2 \%$ en pobreza extrema

En cuanto a la situación educativa de los NNA, el 84,2 \% del grupo de 4 a 17 años de edad asiste a la escuela. La tasa de asistencia escolar es del $84,1 \%$ en niños y $84,4 \%$ en niñas; y por área geográfica se registró una asistencia escolar mayor en el área urbana (88,2 \%) que en el área rural, con el 78,7 \% (EHPM, 2013).

En lo que se refiere al cuido de la niñez y adolescencia salvadoreña, acorde con la EHPM (2013), se observa que la persona que cuida normalmente es la madre $(81,6 \%)$, seguido de los abuelos con el 10,8\%; el 2,7 \% están bajo el cuidado de la empleada, y el $4,9 \%$ es cuidado normalmente por el papá, los tíos, vecinos u otro familiar. Llama la atención que, en los resultados de este estudio, la figura paterna no aparece como uno de los principales cuidadores de este importante grupo poblacional.

\subsection{Conceptualización de maltrato infantil}

Comprender el maltrato infantil que actualmente experimentan muchos NNA, resulta tarea compleja, puesto que, como se mencionó en el apartado anterior, el uso de la violencia como mecanismo de resolución de conflictos ha constituido una práctica históricamente enraizada en la cultura del país (Unicef El Salvador, 2014); situación que se confirma, según Unicef (2014, p. 116), al sostener que "una de las causas ocultas es la práctica de crianza violenta en espacios de socialización de niñas, niños y adolescentes, es decir, en la escuela, en el hogar y en la comunidad".

2 NNA para efectos de este trabajo se entiende por niños, niñas y adolescentes. 
En este contexto, el maltrato infantil es considerado una forma de violencia que puede definirse como "(...) toda acción u omisión que provoque o pueda provocar dolor, sufrimiento o daño a la integridad o salud física, psicológica, moral o sexual de una niña, un niño o adolescente, por parte de cualquier persona, incluidos sus padres madres $u$ otros parientes, educadores y personas a cargo de su cuido (...)" (UTE, 2011, p. 19)

En virtud de lo anterior, el artículo 19 de la convención sobre los derechos del niño, señala que

todas las medidas legislativas, administrativas, sociales y educativas apropiadas para proteger al niño contra toda forma de perjuicio o abuso físico o mental descuido o trato negligente (...) mientras el niño se encuentre bajo la custodia de los padres, de un representante legal o de cualquier otra persona que lo tenga a su cargo (Conna, 2013, p. 359).

Resaltando así la invaluable labor de los padres de familia y cuidadores de los NNA como principales garantes de su protección.

Sin embargo, la praxis cotidiana muestra que la mayor parte de violaciones a los derechos de la niñez y la adolescencia se desarrollan en el ambiente más cercano a la familia, es decir, en el hogar, en la comunidad, en los centros de estudio, espacios en los cuales se desarrolla una serie de maltratos físicos, psicológicos, situaciones de negligencia y alienación parental, los cuales se describen a continuación.

\subsection{Tipos de maltrato}

\section{Maltrato físico}

Soriano $(2015$, p. 1) define el maltrato físico como "toda acción voluntariamente realizada que provoque o pueda provocar daño o lesiones a la integridad física", pudiendo ser empujones, patadas, halones de cabello, etc.

En términos estadísticos, se conoce que 1 de cada 2 niños y niñas experimentan castigo físico en América Latina y el Caribe (Unicef, 2018). A escala mundial, alrededor de 6 de cada 10 niños entre los 2 y 14 años de edad (unos 1.000 millones) sufrieron de manera periódica castigos corporales a manos de sus cuidadores (Unicef, S.f). Los datos confirman que la violencia continúa siendo un problema que requiere mayor análisis.

En el contexto salvadoreño, el fenómeno de la violencia parece no tener mayores diferencias, puesto que en el período 2005-2013 "las Juntas de Protección recibieron 15.035 demandas pertinentes, de las cuales $57,4 \%$ fueron contra la integridad física y $21,5 \%$ por maltrato de niñas, niños y adolescentes (Unicef El Salvador, 2014, p. 20).

La vulnerabilidad de NNA ante situaciones de maltrato queda de manifiesto al considerar que, "de acuerdo con un análisis del Ministerio de Salud con datos de la FESAL 2008, 3 de cada 10 mujeres recibió maltrato físico antes de cumplir 18 años de edad, (Unicef El Salvador, 2014, p. 20).

\section{b) Maltrato psicológico}

El maltrato psicológico constituye otra manifestación de violencia. Comprender su magnitud, se torna una tarea compleja, dado la carencia de indicadores e influencia de la subjetividad en su análisis. Garbarino, Guttman y Seeley (1989) lo definen como un ataque realizado por un adulto sobre el desarrollo de la personalidad y de la competencia social del niño, el cual se manifiesta mediante cinco formas: rechazar, aislar, aterrorizar, ignorar y corromper.

Este planteamiento es compartido por Miranda (2012, p. 81), quien sostiene que, desde la perspectiva de los adultos, "estas actitudes pueden estar dirigidas a dañar la integridad emocional del niño/a través de manifestaciones verbales (...) cuando insultan, rechazan, humillan, desprecian, se burlan, critican, aíslan, atemorizan".

Sin embargo, de manera involuntaria "Ios padres (...) abusan emocionalmente de sus hijos, basados en buenas intenciones, por ejemplo cuando quieren que sobresalgan en el colegio, en el deporte o en la vida social. A partir de esas buenas intenciones pueden presionarlos 0 avergonzarlos al punto de crearles un sufrimiento emocional crónico" (Hernández, 2012, p. 7).

Esta presión, según afirma Miranda (2012, p. 81), puede convertirse en una profunda "desvalorización, baja autoestima e inseguridad personal, frenando el desarrollo social, emocional e intelectual (...) del niño/a". Al respecto, resulta importante considerar cómo los daños emocionales, a consecuencia del maltrato infantil, pueden perdurar durante el resto de la vida (Hernández, 2012). 


\section{c) Negligencia}

La negligencia ejercida por padres, madres o cuidadores es considerada una manifestación de violencia, en el sentido de que "(...) el descuido en el cumplimiento de las obligaciones relativas a la prestación de alimentación nutritiva y balanceada, atención médica, educación o cuidados diarios (...) [(UTE, 2011, p. 20). (Unicef El Salvador, 2014, p. 86)], repercute en el desarrollo integral de la niñez y adolescencia, puesto que la Lepina ${ }^{3}$ establece que "todas las niñas, niños y adolescentes tienen el derecho de gozar de un nivel de vida adecuado en condiciones de dignidad y goce de sus derechos" (Conna, 2013, p. 102).

\section{d) Alienación parental}

El Estado salvadoreño, a través de la Unidad Técnica del sector Justicia, reconoce que "todas las niñas, niños y adolescentes tienen derecho a no ser privados de su libertad, de forma arbitraria o ilegal (...)" (UTE, 2011, p. 20). Asimismo, plantea que "Ias niñas, niños y adolescentes tienen el derecho a mantener con su madre y padre las relaciones afectivas y el trato personal que favorezca el normal desarrollo de su personalidad, aun cuando estén separados, (...)". De esta forma, la privación de todo contacto de los NNA por parte de uno de los progenitores constituye una manifestación de violencia denominada Alienación parental (UTE, 2011, p. 36)

\subsection{Objetivo y justificación del estudio}

Dada la situación de violencia anteriormente descrita, el presente estudio tuvo como objetivo principal identificar situaciones de maltrato infantil en adolescentes salvadoreños que oscilan entre los 13 y 15 años de edad.

Se estima que los resultados que proporcionó el estudio permitirán una comprensión del fenómeno, en la medida que surgió, desde el grupo poblacional que sufre la problemática, siendo este el caso de las y los adolescentes.

\section{Metodología}

Previa reflexión del objetivo de estudio, la investigación fue realizada bajo el paradigma cuantitativo, siendo para ello necesario el uso del método deductivo, el cual, desde un enfoque de género, propició el análisis de las diferentes formas de maltrato experimentadas por adolescentes tanto de género femenino como de masculino.

En congruencia, con el método en mención, se utilizó la técnica de la encuesta por medo de un cuestionario aplicado a 384 adolescentes seleccionados, mediante un muestreo probabilístico, en las tres zonas del país (occidental, central y oriental). La descripción metodológica del estudio se resume en la tabla 1, que se presenta a continuación.

\section{Tabla 1. Ficha técnica de la investigación}

\begin{tabular}{ll}
\hline \multicolumn{1}{c}{ Tipo de estudio } & \multicolumn{1}{c}{ Cuantitativo } \\
\hline Profundidad del estudio: & Descriptivo \\
\hline Técnica de recolección de datos: & Encuesta \\
\hline Instrumento: & Cuestionario estructurado \\
\hline Muestra: & $\begin{array}{l}384 \text { adolescentes de entre } 13 \text { y } 15 \text { años (ubica- } \\
\text { dos en } 7 \text { centros escolares del país) }\end{array}$ \\
\hline Delimitación espacial & 14 departamentos del país \\
\hline Tipo de muestreo: & Probabilístico \\
\hline Nivel de confianza del estudio: & $95 \%$ \\
\hline Margen de error permitido: & $5 \%$ \\
\hline Período de recolección de datos: & Del 8 de mayo al 18 de junio de 2018 \\
\hline Fuente: Elaboración propia &
\end{tabular}

Fuente: Elaboración propia 


\section{Resultados}

Conforme la descripción metodológica expresada en el apartado anterior, a continuación se presentan los resultados del estudio. Al respecto, resulta necesario mencionar que se exploraron indicadores sociodemográficos, manifestaciones de abuso físico, psicológico/emocional; negligencia y alienación parental ejercida en contra de las y los adolescentes, por parte de sus padres y/o cuidadores.

\subsection{Indicadores sociodemográficos}

El universo, para esta investigación, lo constituyó un total de 340.401 adolescentes, cuyas edades oscilaron entre los $13 \mathrm{y}$ 15 años, según el censo escolar del Ministerio de Educación correspondiente al año 2016. Siendo un $51 \%$ de género femenino en correspondencia con un $49 \%$ masculino.

Con la finalidad de garantizar una participación equitativa e igualitaria, tanto masculina como femenina, la selección de los participantes en el estudio, la distribución por edad y sexo, se presenta en el gráfico 1.

\section{Gráfico 1. Adolescentes participantes en el estudio, según edad y sexo}

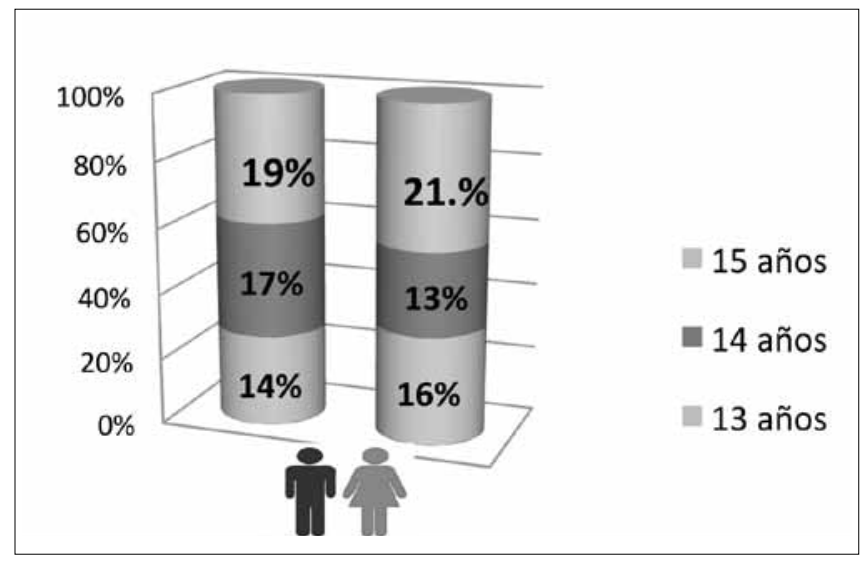

Fuente: Elaboración propia.

Conforme lo expuesto en el gráfico 1, se tuvo la participación de un total de 384 adolescentes, de los cuales 192 fueron de género femenino, correspondiente al $50 \%$ de la muestra seleccionada. Dado la elevada concentración poblacional de adolescentes en la zona central del país, 43 de cada
100 adolescentes fueron de los departamentos de La Paz y San Salvador.

En lo que se refiere a la variable edad, el estudio propició la participación de jóvenes entre los 13 y 15 años de edad. Sin embargo, dado la situación de adolescentes de 15 años con sobreedad, ${ }^{4}$ en muchos centros escolares del país, se registró un $19 \%$ de representación para el género masculino y $21 \%$ en lo que refiere al género masculino.

Asimismo, con el propósito de propiciar representatividad en la selección de la muestra, y por lo consiguiente en los resultados del estudio, se planificó la participación de adolescentes residentes en las tres zonas del país (occidental, central y oriental). En ellas, se seleccionaron siete centros escolares ubicados en seis departamentos, según se muestra en la figura 1.

\section{Figura 1. Ubicación de centros escolares participantes en el estudio}

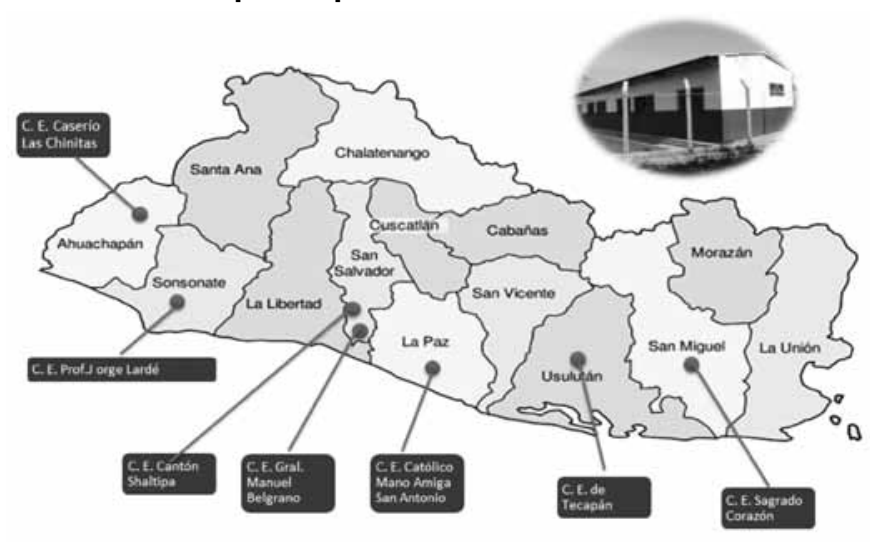

Fuente: Elaboración propia, a partir de Googleimagenes.com.

La figura 1, indica que el estudio posibilitó la participación de las tres zonas del país. Muestra de ello es que, por ejemplo, en la zona occidental se aplicó la encuesta a 95 adolescentes (54 hombres y 41 mujeres) provenientes del Centro Escolar Prof. Jorge Lardé (Sonsonate) y del Centro Escolar Caserío Las Chinitas (Ahuachapán).

En la zona central, fue posible encuestar a 166 adolescentes (71 hombres y 95 mujeres) de tres instituciones educativas, siendo estas: Centro Escolar Cantón Shaltipa, Centro Escolar General Manuel Belgrano (ambos de San Salvador) y Complejo Educativo Católico Mano Amiga San Antonio (La Paz).

4 Puede considerarse como un desfase entre la edad cronológica y la edad escolar; es decir, que existe sobreedad escolar cuando la edad cronológica está por encima del grado o nivel académico alcanzado. 
Finalmente, en la zona oriental, se logró la participación de dos centros educativos: Complejo Educativo de Tecapán (Usulután) y Centro Escolar Sagrado Corazón (San Miguel), ambas instituciones posibilitaron la participación de 123 adolescentes (67 hombres y 56 mujeres) entre las edades de 13 y 15 años.

\subsection{Abuso físico}

En términos generales, "se reconoce el rol fundamental de la familia como medio natural para garantizar la protección integral de las niñas, niños y adolescentes; y su papel primario y preponderante en la educación y formación de los mismos" (UTE, 2011, p. 6). Sin embargo, en reiteradas ocasiones, los golpes o heridas físicas generadas a NNA evidencian una realidad que experimenta este grupo poblacional, según lo manifestaron los adolescentes, y que se representa en el gráfico 2.

Gráfico 2. Durante el último año, ¿algún adulto a cargo de tu cuidado, te lastimó físicamente?

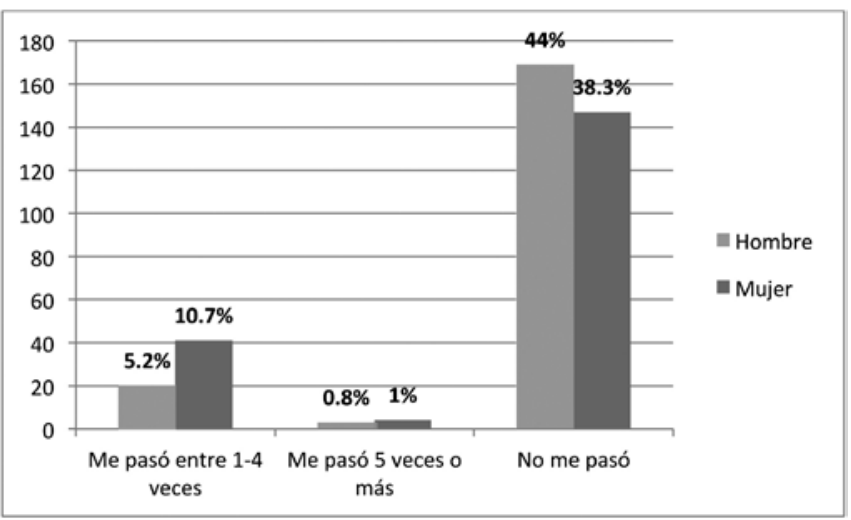

Fuente: Elaboración propia.

Conforme lo expuesto en el gráfico 2, un $17,7 \%$ de adolescentes salvadoreños ha sufrido maltrato físico durante el último año, es decir, 1 de cada 6 adolescentes ha sido víctima de este tipo de violencia. Un aspecto que se debe destacar es que este porcentaje resultó inferior al promedio de América Latina y el Caribe, que es del $50 \%$, en el cual 1 de cada 2 niños y niñas experimentan castigo físico en su vida (Unicef, 2018).

Por otra parte, los resultados muestran que esta forma de violencia ha sido identificada en mayores proporciones por el género femenino (11,7\%) que el masculino (5,3\%).

\subsection{Abuso psicológico/emocional}

El Estado salvadoreño señala que "todo menor tiene derecho a vivir en condiciones familiares (...) que le permitan su desarrollo integral, para lo cual tendrá la protección del Estado" (Constitución de la República de El Salvador, 1983) y que a su vez "el niño debe, en todas las circunstancias, figurar entre los primeros que reciban protección (...)" (Conna, 2013, p. 347).

Lograr este cometido, no resulta tarea sencilla, puesto que muchas prácticas de crianza, consciente 0 inconscientemente, se convierten en manifestaciones de abuso psicológico/emocional, siendo ejemplo de ello el sentirse mal porque "un cuidador" insulta, desprecia o dice palabras vulgares a niños, niñas y adolescentes. Bajo esta consideración, el estudio encontró que los adolescentes han experimentado situaciones de violencia emocional, según se expone en el gráfico 3.

Gráfico 3. Durante el último año, ¿te asustaste o te sentiste mal porque los adultos a cargo de tu cuidado te insultaban, te decían cosas vulgares o porque no te querían?

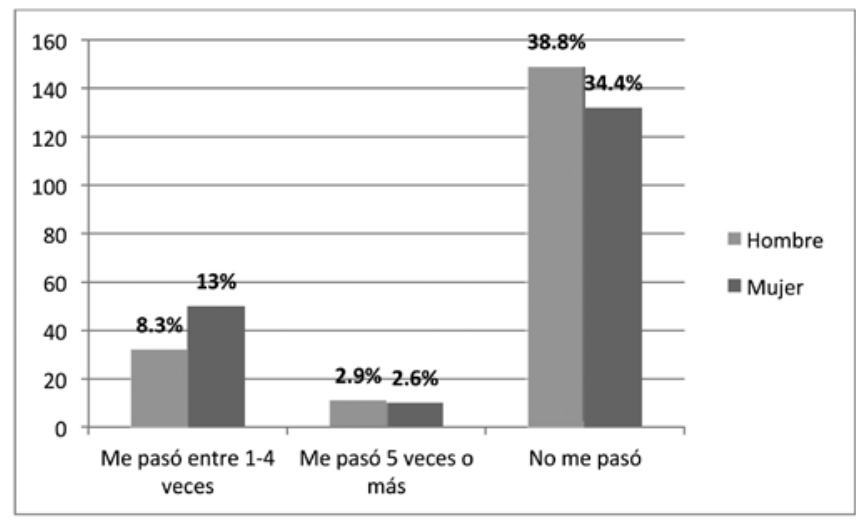

Fuente: Elaboración propia.

El $26,8 \%$ de los adolescentes reconocen haber sufrido abuso psicológico/emocional por parte de sus padres o cuidadores durante el año 2018. Los resultados indican que adolescentes de género femenino $(15,6 \%)$ han experimentado mayores situaciones de violencia de este tipo en relación con los hombres (11,2\%), quienes en su mayoría $(38,8 \%)$ afirman no haber tenido experiencias que denoten este tipo de abuso o maltrato. Tal afirmación, desde la perspectiva masculina, puede interpretarse como efecto de la influencia de la cultura patriarcal impregnada en la sociedad salvadoreña. 


\subsection{Negligencia}

La negligencia constituye otra forma de violencia ejercida contra la niñez y adolescencia. Se manifiesta en la medida que los padres de familia no dan los cuidados necesarios a un niño, niña o adolescente para un buen desarrollo. Es decir, el descuido de alimentación, salud, educación, recreación, entre otras, indica la existencia de este tipo de violencia, que opera de manera silenciosa.

Otro aspecto por resaltar es el limitado reconocimiento de la negligencia como mecanismo de violencia, lo cual evidencia que es muy poco percibido. Esta afirmación, se argumenta con la opinión de los adolescentes, conforme se detalla en el gráfico 4.

\section{Gráfico 4. Durante el último año, ¿tus padres fueron descuidados contigo?}

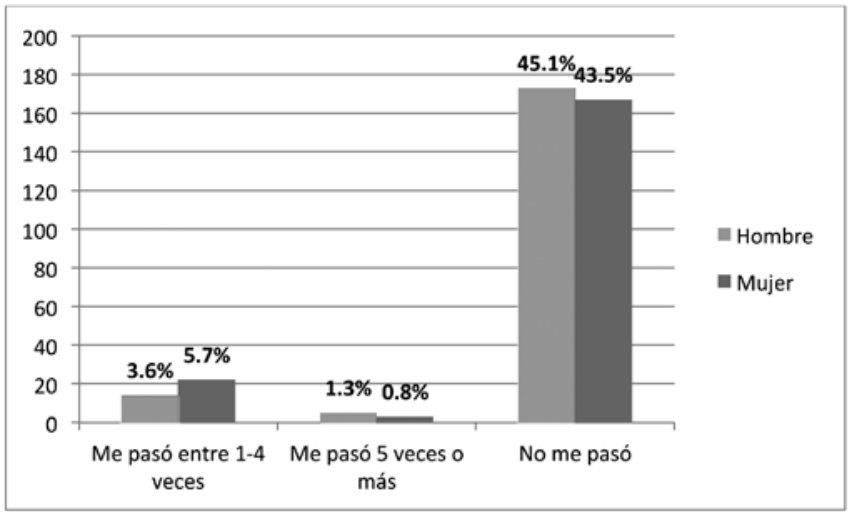

Fuente: Elaboración propia.

Según los adolescentes salvadoreños, un $11,4 \%$ de ellos ha sufrido trato negligente de parte de sus padres 0 cuidadores durante el año 2018. Como puede observarse, adolescentes de género femenino (6,5\%) manifiestan haber experimentado este tipo de violencia, a diferencia de adolescentes de género masculino (4,5\%).

Un aspecto que se ha de considerar en el análisis de los resultados presentados es que este tipo de violencia opera de manera oculta y silenciosa, y por eso suele ser muy poco reconocida por la población en general, a pesar de su gran impacto en el desarrollo de todo ser humano. Este estudio indica que 1 de cada 9 adolescentes ha sido víctima de negligencia por parte de sus padres o cuidadores.

\subsection{Alienación parental}

La alienación parental fue el último tipo de violencia explorado en esta investigación, básicamente, el cual consiste en obstaculizar o privar el derecho del niño, niña o adolescente de convivir y tener cercanía con el otro progenitor.

El estudio señala que, a pesar de que el Estado salvadoreño, a través de la Unidad Técnica del sector Justicia, reconoce que "todas las niñas, niños y adolescentes tienen derecho a no ser privados de su libertad, de forma arbitraria 0 ilegal (...)" (UTE, 2011, p. 20), existen casos que indican lo contrario, según se muestra en el gráfico 5.

Gráfico 5. Durante el último año, ¿alguno de tus padres te llevó a otro lugar o te escondió para que no vieras o estuvieras con el otro?

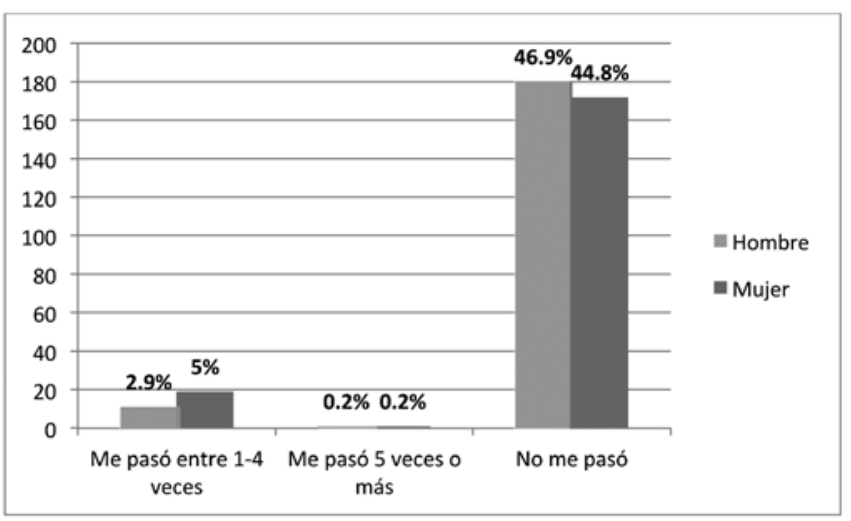

Fuente: Elaboración propia.

Según lo expresado en el gráfico, el 8,3\% de los adolescentes de El Salvador afirman que uno de sus progenitores les impidió, sin justificación alguna, poder estar con otro de sus parientes cercanos, es decir, en el año 2018, 1 de cada 13 adolescentes consideraron haber sido víctimas de alienación parental.

Así mismo, los resultados indican que las adolescentes continúan siendo las afectadas por este tipo de violencia $(5,2 \%)$ en relación con adolescentes masculinos $(3,1 \%)$, pese a la amplia existencia de marcos normativos que velan por la protección de la niñez y la adolescencia.

Finalmente, al haber indagado cuatro situaciones de maltrato infantil, siendo estas maltrato físico, abuso psicológico, 
negligencia y alienación parental, se encontró que las dos primeras resultaron ser las principales manifestaciones de violencia experimentadas por adolescentes, según se observa en el gráfico 6.

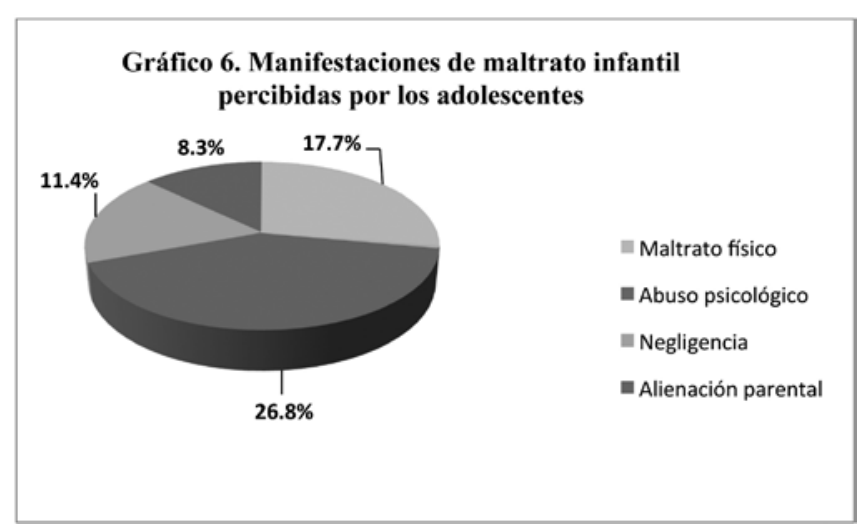

Fuente: Elaboración propia.

\section{Conclusiones}

En congruencia con el objetivo de investigación y los resultados expuestos en el apartado anterior, a continuación se enuncian las conclusiones del estudio.

1. El estudio confirma que la niñez y la adolescencia salvadoreña son víctimas de maltrato infantil, conforme al promedio mundial señalado por Unicef (2018), al encontrar que 1 de cada 2 adolescentes (64\%), de entre 13 y 15 años de edad, ha experimentado por lo menos una situación de maltrato infantil por parte de sus padres o cuidadores, durante el año 2018.

2. El maltrato hacia la niñez y adolescencia, continúa siendo una forma de violencia ejercida con mayor proporción y frecuencia en perjuicio de las mujeres, siendo esto un reflejo de la incidencia de la cultura patriarcal, según muestra el estudio, luego de descubrir que de cada 3 adolescentes que sufrieron violencia física, 2 de ellas resultaron ser de género femenino.

3. Durante el último año, el abuso psicológico/emocional $(26,8 \%)$ y maltrato físico $(17,7 \%)$ son las principales manifestaciones de violencia experimentadas por los adolescentes, tanto masculinos como femeninos, seguido de la negligencia $(11,4 \%)$ y alienación parental $(8,3 \%)$. En este sentido, se presume que el limitado porcentaje obtenido de las dos últimas obedece al alto nivel de desconocimiento y claridad conceptual de estas por parte de la sociedad salvadoreña en general.

\section{Referencias}

CONNA. (2013). Recopilación de normativa de niñez y adolescencia en El Salvador. San Salvador, El Salvador: Contracorriente editores.

DIGESTYC. (2013). Resultados encuesta de hogares de propósitos múltiples 2013 [versión de Adobe Acrobat Reader]. Recuperado de http://www.digestyc.gob.sv/ EHPM2013/ DIGESTYC/presentacion.pdf

Garbarino, J., Guttman, E. y Seeley, J. (1989). The psychologically battered child. Strategies for identification, assessment and intervention (4ta Ed.). San Francisco, EE.UU: Jossey-Bass Inc.

Hernández Miranda, J. E. (2012). Características psicosociales de niños/as que experimentan maltrato infantil de 7-9 años del $1^{\circ}$ ciclo de la Escuela Dr. José Rosa Pacas, del municipio de San Vicente, departamento de San vicente en el período comprendido de mayo 2010 a septiembre 2012 (Tesis de pregrado). Universidad de El Salvador, El Salvador.

Soriano Faura, F. J. (2015). Promoción del buen trato y prevención del maltrato en la infancia en el ámbito de la atención primaria de la salud [versión de Adobe Acrobat Reader]. Recuperado de http://previnfad. aepap.org/sites/default/files/2017-04/previnfad_ maltrato. pdf

Unicef. (2014). Informe de situación de la niñez y adolescencia en El Salvador 2014: Transformar inequidades en oportunidades para todas las niñas, niños $y$ adolescentes. Recuperado de https://www.unicef.org/ elsalvador/informes/informe-de-situaci\%C3\%B3n-dela-ni\%C3\%B1ez-y-adolescencia-en-el-salvador-2014

Unicef. (2018). Disciplina violenta en América Latina y el Caribe: Un análisis estadístico 2018. Recuperado de https://www.unicef.org/lac/informes/disciplinaviolenta-en-am\%C3\% A9rica-latina-y-el-caribe

Unicef. (s.f.). Ocultos a plena luz: Un análisis estadístico de la violencia contra los niños [versión de Adobe Acrobat Reader]. Recuperado de https://www.unicef.org/ ecuador/ocultos-a-plena-luz.pdf

Unidad Técnica Ejecutiva. (2011). Ley de Protección Integral de la Niñez y Adolescencia (LEPINA). Recuperado de http://www.ute.gob.sv/index.php/tema/2014-12-0101-40-25/2019-01-16-21-52-11/lepina.html 